\title{
Case report 725
}

\section{Leslie A. Welk, M.D. and Ronald S. Adler, M.D., Ph.D.}

Department of Radiology, University of Michigan Hospital, Ann Arbor, Michigan, USA
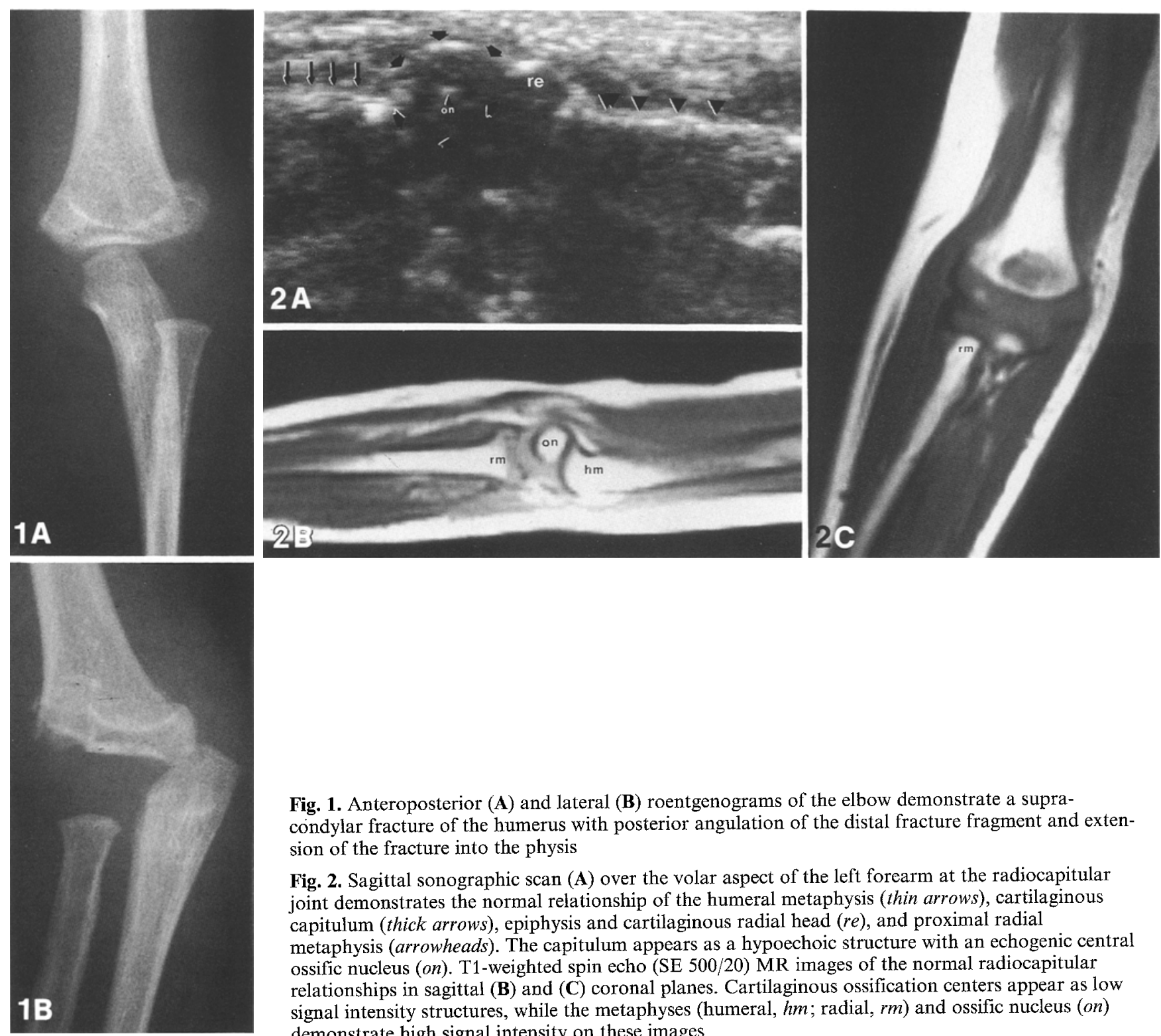

Fig. 1. Anteroposterior (A) and lateral (B) roentgenograms of the elbow demonstrate a supracondylar fracture of the humerus with posterior angulation of the distal fracture fragment and extension of the fracture into the physis

Fig. 2. Sagittal sonographic scan (A) over the volar aspect of the left forearm at the radiocapitular joint demonstrates the normal relationship of the humeral metaphysis (thin arrows), cartilaginous capitulum (thick arrows), epiphysis and cartilaginous radial head (re), and proximal radial metaphysis (arrowheads). The capitulum appears as a hypoechoic structure with an echogenic central ossific nucleus (on). T1-weighted spin echo (SE 500/20) MR images of the normal radiocapitular relationships in sagittal (B) and (C) coronal planes. Cartilaginous ossification centers appear as low signal intensity structures, while the metaphyses (humeral, $\mathrm{hm}$; radial, $\mathrm{rm}$ ) and ossific nucleus (on) demonstrate high signal intensity on these images 


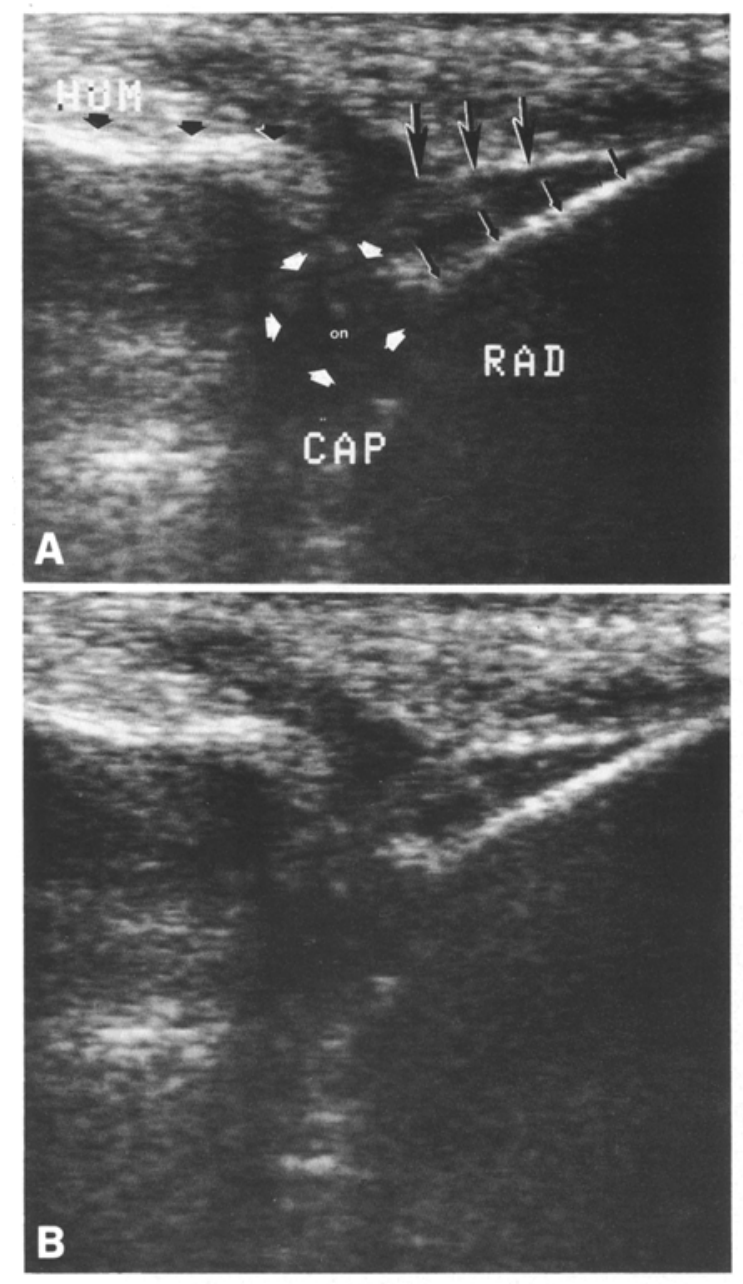

Fig. 3. Sagittal sonographic scan (A) over the volar aspect of the right forearm at the radiocapitular joint reveals posterior displacement of the radius $(R A D$, thin black arrows), and capitulum (CAP, short white arrows) as a unit relative to the distal humerus (HUM, thick black arrows). The echogenic capitulum ossific nucleus (on) is helpful in locating the capitulum and in demonstrating its relationship to the adjacent proximal radius. A complex joint effusion appears as low-level echoes situated between the displaced capsule (large black arrows) and radial metaphysis (thin black arrows). An unlabeled image is provided for comparison (B)

\section{Clinical information}

An 11-month-old boy presented to the Emergency Room with a swollen,

Address reprint requests to: Ronald S. Adler, M.D., Ph.D., University of Michigan Medical Center, 1500 E. Medical Center Drive, Ann Arbor, MI 48109-0030, USA painful right elbow. A plain film radiographic examination performed in the anteroposterior and lateral projections demonstrated a supracondylar fracture of the humerus with posterior angulation of the distal fracture fragment and extension of the fracture line into the physis (Fig. 1). Evaluation for dislocation and involvement of the distal humeral growth plates was difficult, secondary to lack of ossification of the secondary centers of ossification. Real-time ultrasonography of both elbows was subsequently performed using an Acuson 128 unit and a 5$\mathrm{MHz}$ linear phased array transducer with scans performed in sagittal and transverse planes. This revealed a normal relationship of the asymptomatic left humerus, capitulum, and radius (Fig. 2A). For purposes of i1lustration of the anatomic relationships, T1-weighted contrast (SE 500/ 20), sagittal and coronal magnetic resonance (MR) images (GE Signa 1.5-T unit) of the normal radiocapitular joint are presented in another child (Fig. 2B, C). Sonographic examination of the right elbow demonstrated a complex joint effusion, appearing as low-level echoes within the enlarged joint space, and a supracondylar fracture of the humerus identified as a discontinuity in the cortical bone of the distal humeral shaft. The proximal end of the right radius and capitulum were posteriorly displaced relative to the distal end of the humerus, while the radiocapitular joint was maintained, indicating a displaced fracture rather than a fracture dislocation of the elbow (Fig. 3).

The patient subsequently underwent closed reduction with percutaneous pin fixation of the supracondylar fracture and was placed in a long arm cast. The cast and pins were removed approximately 1 month later, at which time the patient possessed good motor and sensory function of the right forearm. 
Diagnosis: Posterior displacement of proximal end of the right radius and capitulum with supracondylar fracture causing a displaced fracture rather than a fracture dislocation

\section{Discussion}

Fractures of the distal end of the humerus usually pose no diagnostic problem in adults and are readily evaluated on the anteroposterior and lateral plain film projections of the elbow. In children, on the other hand, diagnosis may be problematic due to the presence of the predominantly cartilaginous secondary centers of ossification and their variability in appearance. The normal ages of radiographic appearance of the four secondary ossification centers of the distal humerus are capitulum at 1-2 years, medial epicondyle at 4 years, trochlea at 8 years, and lateral epicondyle at 10 years [6]. Evaluation by plain film radiography of the distal humeral epiphysis is difficult prior to ossification of the secondary ossification centers, as it relies upon displacement of these centers as an indicator of physeal disruption. Fractures involving the distal humeral epiphysis may be misdiagnosed as a dislocated elbow, particularly in infants and young children. The most important distinguishing feature is the normal relationship of the ossification center of the capitulum to the proximal end of the radius. This relationship may not be readily evident in the small child. It is important to distinguish between the two entities in order to institute proper therapy. Dislocation of the elbow is commonly treated with closed reduction alone, whereas distal humeral epiphyseal separation may require percutaneous (pin) fixation or open reduction [8]. In the past, arthrography was utilized to evaluate the extent of physeal involvement [5]. Because of its ability to visualize the cartilaginous secondary ossification centers, ultrasonography may prove to be a useful tool in the evaluation of suspected fractures and dislocations in young children. The rapid, noninvasive nature of this modality also makes it especially attractive.

Ultrasonic energy in the therapeutic range has been used to confirm and/or diagnose fractures $[1,4,9]$. Although its mode of action is unclear, ultrasonic energy in this range is known to be capable of producing pain or severe tingling when applied to fractures less than 2 weeks old. Recently, real-time sonography has proven useful in the diagnosis of fractures of the clavicle in the newborn [6] and occult bone fractures in infants [5].

The bone-soft tissue interface forms a strong specular reflector due to the large differences in acoustic impedance. As a result, the underlying cancellous bone is not well evaluated since the majority of the acoustic energy is reflected [5]. Nevertheless, the bone contour can be examined, and in our case this demonstrated the presence of a supracondylar fracture as evidenced by cortical disruption along the medial and lateral supracondylar ridges of the distal humerus. In addition, ultrasound demonstrates the cartilaginous ossification centers appearing as hypoechoic structures with the central ossific nuclei appearing as small, bright, echogenic foci, allowing for evaluation of their relationship to subjacent cartilaginous and bony structures [7]. Displacement of the capitulum and proximal end of the radius in this case signified displacement without dislocation. Evaluation of the physis is also possible using ultrasound, although this issue was not specifically addressed in this case.

Sonography can provide useful complementary information to the routine radiographic examination of suspected fractures of the skeleton, particularly with regard to evaluation of bony contour, disruption of the physis, and maintenance of articular relationships $[2,3]$. These fea- tures are especially true in younger patients in whom lack of ossification of the secondary ossification centers limits plain film analysis.

In summary, an 11-month-old boy with a swollen, painful right elbow demonstrated a supracondylar fracture on plain film radiographic studies. Sonography was subsequently performed to exclude dislocation of the elbow and demonstrated the supracondylar fracture as well as a normal relationship of the capitulum and radius, signifying a displaced fracture without associated dislocation. A bone dislocation would generally require dosed reduction alone, whereas distal humeral epiphyseal separation usually requires open reduction or percutaneous (pin) fixation. This case demonstrates the utility of ultrasound in the evaluation of physeal disruption and dislocation in the elbow prior to ossification of the secondary centers of ossification.

\section{References}

1. Dacruz D, et al. (1988) Ultrasound assessment of the suspected scaphoid fracture. Arch Emerg Med 5(2):97

2. Dias JJ, Lamont AC, Jones JM (1988) Ultrasonic diagnosis of neonatal separation of the distal humeral epiphysis. $J$ Bone Joint Surg [Br] 70:825

3. Dorr U, Zieger M, Hauke H (1988) Ultrasonography of the painful hip: prospective studies in 204 patients. Pediatric Radiol 19:36

4. Giladi M, et al. (1984) Comparison between radiographs, bone scan, and ultrasound in the diagnosis of stress fractures. Milit Med 149(8):459

5. Graif M, et al. (1988) Sonographic detection of occult bone fractures. Pediatr Radiol 18(5):383

6. Greenspan A (1988) Orthopedic radiology. J.B. Lippincott, Philadelphia, p 3.23

7. Harcke HT, Lee MS, Sinning L (1986) Ossification center of the infant hip: sonographic and radiographic correlation. AJR 147:317

8. Ogden J (1990) Skeletal injury in the child. W.B. Saunders, Philadelphia, p 387

9. Shenouda N, England J (1987) Ultrasound in the diagnosis of scaphoid fractures. J Hand Surg 12B:43 